\title{
Influenza vaccination is safe and effective in patients suffering from fibromyalgia syndrome
}

\author{
J.N. Ablin', V. Aloush1', A. Brill'1, M. Berman'1, M. Barzilai², \\ D. Caspi', M. Mandelboim³, D. Levartovsky¹, A. Polachek', Y. Wolman', \\ D. Paran ${ }^{1}$, M. Barkagan ${ }^{4}$, O. Elkayam ${ }^{1}$ \\ ${ }^{1}$ Institute of Rheumatology, Tel Aviv Sourasky Medical Center, \& Sackler School of Medicine, Tel Aviv \\ University, Tel Aviv, Israel; 'Institute of Hematology, Tel Aviv Sourasky Medical Center, Israel; \\ ${ }^{3}$ Sheba Medical Center, Tel Aviv, Israel; ${ }^{4}$ nstitute of cardiology, Tel Aviv Sourasky Medical Center, Israel
}

\begin{abstract}
SUMMARY
The fibromyalgia syndrome (FMS) is considered to result from the exposure of a genetically susceptible individual to various triggers, such as physical trauma, stress, viral infections etc. A possible role of vaccination in FMS etiology has been suspected. Our objective was to evaluate the efficacy and safety of influenza vaccination in FMS patients.

Nineteen FMS patients underwent physical and dolorimetric examinations and answered the fibromyalgia impact questionnaire (FIQ), the widespread pain index (WPI) checklist and the symptoms severity scale (SSS), which are part of the 2010 diagnostic criteria. Thirty-eight healthy subjects were recruited as controls. All participants were vaccinated with the inactivated split virion influenza vaccine. Serum was collected for antibody titration. Six weeks after vaccination, sera were tested by hemagglutination (HI) against A/California (H1N1), $\mathrm{A} /$ Perth (H3N2) and B/Brisbane. Humoral response was defined as either a fourfold or greater increase in titer, or an increase from a non-protective baseline level of $<1 / 40$ to a level of $1 / 40$.

No severe vaccination reactions were observed. No significant change was observed between WPI, SSS and FIQ values before and after vaccination, indicating no worsening of FMS symptoms.

Vaccine immunogenicity: Six weeks after vaccination, FMS patients showed a significant increase in geometric mean titers of $\mathrm{HI}$ antibody. The rates of sero-protection increased from $22.9 \%$ for $\mathrm{H} 1 \mathrm{~N} 1$ to $89.5 \%$ post-vaccination. A significant increase in HI antibody titers was also demonstrated among healthy controls. Influenza vaccination was both safe and effective in FMS patients. In view of these results, FMS patients should be encouraged to undergo influenza vaccination according to the standard WHO recommendations.
\end{abstract}

Key words: Fibromyalgia; influenza vaccination.

Reumatismo, 2015; 67 (2): 57-61

\section{INTRODUCTION}

$\mathrm{F}$ ibromyalgia is a clinical syndrome characterized by chronic widespread pain with tenderness and fatigue. Central sensitization is considered a major pathogenic feature of the fibromyalgia syndrome (FMS) (1). While the etiology of FMS is not yet completely understood, it is generally considered to result from the interaction between a specific genetic background and word the exposure of a susceptible individual to various enhancing triggers, such as physical trauma, infection, stress, etc. (2). The potential role of vaccination in causing or exacerbating FMS had already been suspected in the past $(3,4)$. In view of this background it is of considerable clinical importance to ascertain both the efficacy and safety of vaccination in patients suffering from FMS.

Our group has previously established that influenza vaccination is safe and effective in rheumatoid arthritis patients (5). Therefore, the aim of this study was to ascertain its efficacy and safety in patients suffering from FMS.

\section{MATERIALS AND METHODS}

Nineteen patients were recruited in a specialized FMS clinic. Inclusion criteria were
Corresponding author: Jacob N. Ablin

Tel Aviv Sourasky Medical Center Tel Aviv, Israel

E-mail: ajacob@post.tau.ac.il 
prior diagnosis of fibromyalgia, age over 18 and ability to give informed consent. Exclusion criteria were a known allergy to egg products or influenza vaccine, a febrile disease in progress and pregnancy.

Each patient reported his/her complete history including the use of medications, and underwent physical examination and dolorimetry before undergoing vaccination. The fulfillment of the American College of Rheumatology (ACR) 1990 FMS classification criteria was duly documented (6). Patients filled in the widespread pain index (WPI) checklist and the symptoms severity scale (SSS), derived from the 2010-2011 ACR preliminary diagnostic criteria for FMS $(7,8)$ and the Hebrew version of the fibromyalgia impact questionnaire (FIQ) (9). Healthy controls (38) were recruited from the hospital staff. The study was approved by the institutional ethics committee and all patients gave a written informed consent.

Participants were vaccinated with the inactivated split virion influenza vaccine, recommended by the WHO and serum samples were collected for antibody titration. Six weeks after vaccination, patients were evaluated again and serum was collected for the second time. Patients were also asked whether they developed any direct vaccine-related side effects and how were their general health conditions following vaccination.

\section{Immunogenicity of the vaccine}

The pre- and post-immunization hemagglutination (HI) antibodies were tested at the Central Virology Laboratory of the Israeli Ministry of Health using the HI test according to the standard WHO procedure (10). Sera were tested by HI test against the three antigens included in the vaccine: $\mathrm{A} /$ California (H1N1), A/Perth (H3N2) and B/Brisbane. Sera were separated, code labeled, and stored at $-20^{\circ} \mathrm{C}$ until the test was conducted. They were treated with receptor-destroying enzyme cholera filtrate to remove non-specific inhibitors, and with Turkey red blood cells to remove non-specific agglutinins. The treated sera were tested by an $\mathrm{HI}$ test against the three antigens included in the vaccine: $\mathrm{A} /$ California (H1N1), A/Perth (H3N2) and B/ Brisbane. The effective dilution (test dose) of each antigen contained 4 HA units in 25 $\mu \mathrm{L}$ of antigen. Test doses were diluted in phosphate-buffered saline and added to serial dilutions of anti-serum. The HA inhibition titer was determined as the highest dilution of serum that inhibited completely the hemagglutination of red blood cells. Humoral response was defined as either a fourfold or greater increase in titer, or a rise from a non-protective baseline level of $<1 / 40$ to $1 / 40$ after vaccination. The titer of an antiserum that did not show any inhibition was recorded as 1/10. Geometric mean titers of antibodies were calculated to assess the entire group immunity.

\section{Statistical methods}

Non-parametric tests were used in the analysis, since most parameters were not normally distributed. In addition, parametric tests were performed for the log transformation of the parameters. The statistical analysis was performed using the SPSS system for Windows, release 17.0.

\section{RESULTS}

No severe vaccination-related events were documented among patients or controls enrolled in this study.

\section{Characteristics of patients and controls}

Nineteen FMS patients and 38 healthy controls were recruited. Sixteen out of nineteen patients $(84.2 \%)$ were female and their mean age was 46.6 years.

\section{Effect of vaccination against influenza on disease activity}

Vaccination against influenza was not associated with a significant worsening of any clinical index of disease activity (Table I). As shown in the table, no significant change was observed between WPI, SSS and FIQ values before and after vaccination, indicating no resulting clinical change. There was no local reaction to vaccination in any group. No severe vaccination reactions were observed. 
Table I - Clinical features of fibromyalgia before and after influenza vaccination.

\begin{tabular}{|l|c|c|c|}
\hline & Pre vaccination & Post vaccination & $\mathbf{p}$ \\
\hline WPI (DS) & $13,3(4,7)$ & $11(5,4)$ & 0,19 \\
\hline SSS (DS) & $9,1(1,6)$ & $9,0(2,7)$ & 0,88 \\
\hline FIQ (DS) & $67,3(14,2)$ & $65,9(22,0)$ & 0,82 \\
\hline
\end{tabular}

The above values are means standard deviation (SD). WPI, widespread pain index; SSS, symptom severity score; FIQ, fibromyalgia impact questionnaire.

\section{Immunogenicity of the vaccine}

Six weeks after vaccination, FMS patients displayed a significant increase in the geometric mean titers of the $\mathrm{HI}$ antibody against $\mathrm{H} 1 \mathrm{~N} 1$ and $\mathrm{B} /$ Bri viruses: from 29.9 to $387.9(\mathrm{p}=0.0011)$, from 82.9 to 460.9 $(\mathrm{p}=0.0007)$ respectively. The increase for Perth virus was borderline from 28.8 to $96.0(\mathrm{p}=0.08)$. The rates of sero-protection (defined as antibody levels above 1/40) increased from $22.9 \%$ for $\mathrm{H} 1 \mathrm{~N} 1$ to $89.5 \%$ post vaccination (Figure 1).

\section{DISCUSSION}

This study has demonstrated that influenza vaccinations are both safe and effective in patients suffering from fibromyalgia. This conclusion is of significant practical importance for health care providers who need to inform their FMS patients about risks and benefits of vaccination.

As noted above, the potential link between FMS and prior exposure to vaccines is an issue which has raised significant interest and debate in the literature. Not only was this discussion focused on fibromyalgia per se, but also on the broader field of central-sensitization-related conditions as well as other multi-system and enigmatic conditions. For instance, the Gulf war syndrome, an entity with considerable clinical overlap with FMS, was considered to be potentially linked with exposure to multiple vaccinations $(11,12)$. More recently, a theory has been proposed regarding the possibility that vaccination-related adjuvants may induce a multi-system disorder named autoimmune syndrome induced by adjuvants (ASIA), characterized by symptoms such

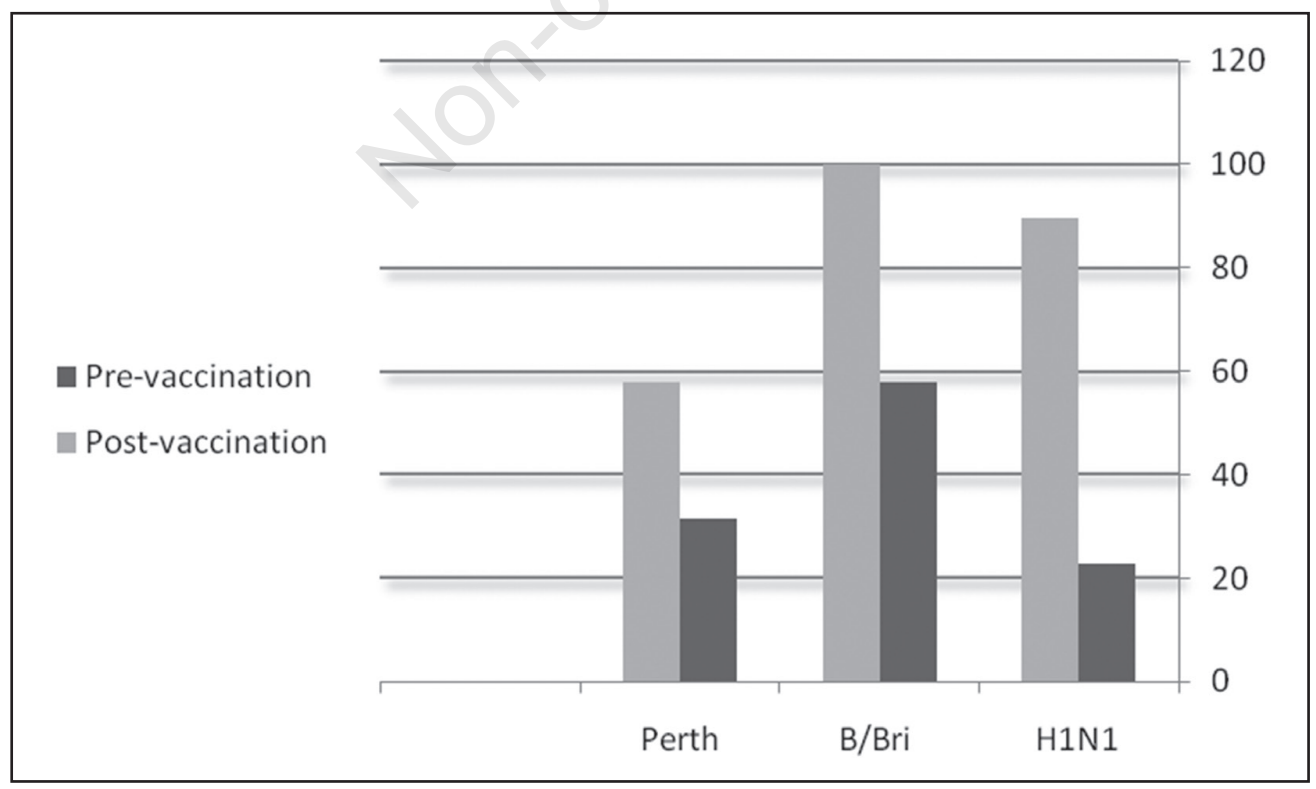

Figure 1 - Change in geometric mean titers $(\mu \mathrm{g} / \mathrm{mL})$ of hemagglutination antibody against influenza antigens before and 6 weeks after vaccination. 
as fatigue, cognitive impairment and arthragia $(13,14)$. Both the ASIA syndrome and FMS have recently been reported to be associated with hepatitis B vaccination (15). Moreover, the Gulf war syndrome was also suggested to be a particular type of this syndrome (16). The chronic fatigue syndrome (CFS), another condition with considerable overlap with FMS, was also linked with vaccination in the past (17).

The postural orthostatic tachycardia syndrome, another clinical entity which overlaps CFS, has also recently been noted in association with vaccination (18).

However, as previously mentioned, infections per se are considered one of the potential etiological triggers for the development of FMS (19-22). Hence, avoiding vaccination is not without consequences for FMS patients, who may then be at an increased risk of contracting various infections, both viral and non-viral. The actual impact of such infections on the course of FMS patients (or individuals who may be prone to develop FMS) is hard to estimate. However, from a practical point of view, physicians treating FMS patients are frequently confronted with the question of administering the vaccine or not. Also, many patients may choose to avoid vaccination given the actual implications are still unclear. A recent study conducted on the population of Madrid (Spain) demonstrated extremely low rates of vaccination against influenza (14.2\%) among FMS patients (23).

However, since influenza continues to be a major public health issue in many countries, these results underlie the importance of ascertaining the safety of vaccination in specific patient populations.

Another aspect addressed by our study is the immunological efficacy of vaccination in FMS patients. While FMS is not regarded as an autoimmune disorder and is not regularly treated with medications capable of suppressing the activity of the immune system, various subtle aspects that are typical of immune dysfunctions have frequently been documented among this patient population (24-26). Therefore, the demonstration given by this study that
FMS patients can be immunized successfully against influenza is another significant outcome.

However, it is worth pointing out that this study has some limitations. The small number of participants is an obvious limitation, although, due to its exploratory nature, we consider it to be still noteworthy. Future studies should ideally investigate the relationship between vaccination of healthy pain-free individuals, and the prospective development of FMS symptoms, which has not yet been reported in the literature.

\section{CONCLUSIONS}

Influenza vaccination was safe and effective in FMS patients. No clinical change was reported following vaccination and serological evidence of successful vaccination was similar to that observed among healthy controls.

In view of these results, FMS patients should be encouraged to undergo influenza vaccination according to the standard WHO recommendations.

Key points: Despite debate in the literature regarding the role of vaccinations in many connective tissue disorders, vaccinating FMS patients against influenza is both safe and effective.

\section{REFERENCES}

1. Yunus MB. Fibromyalgia and overlapping disorders: the unifying concept of central sensitivity syndromes. Semin Arthritis Rheum. 2007; 36: 339-56.

2. Ablin JN, Cohen H, Buskila D. Mechanisms of disease: genetics of fibromyalgia. Nat Clin Pract Rheumatol. 2006; 2: 671-8.

3. Ablin JN, Shoenfeld Y, Buskila D. Fibromyalgia, infection and vaccination: two more parts in the etiological puzzle. J Autoimmun. 2006; 27: $145-52$.

4. Cassisi G, Sarzi-Puttini P, Cazzola M. Chronic widespread pain and fibromyalgia: could there be some relationships with infections and vaccinations? Clin Exp Rheumatol. 2011; 29: S118-26.

5. Elkayam O. Safety and efficacy of vaccination against influenza in patients with rheumatoid arthritis. Clin Dev Immunol. 2006; 13; 34951. 
6. Wolfe F, Smythe HA, Yunus MB, Bennett RM, Bombardier C, Goldenberg DL, et al. The American College of Rheumatology 1990 criteria for the classification of fibromyalgia. Report of the Multicenter Criteria Committee. Arthritis Rheum. 1990; 33: 160-72.

7. Wolfe F, Clauw DJ, Fitzcharles MA, Goldenberg DL, Katz RS, Mease P, et al. The American College of Rheumatology preliminary diagnostic criteria for fibromyalgia and measurement of symptom severity. Arthritis Care Res (Hoboken). 2010; 62: 600-10.

8. Wolfe F, Clauw DJ, Fitzcharles MA, Goldenberg DL, Häuser W, Katz RS, et al. Fibromyalgia criteria and severity scales for clinical and epidemiological studies: a modification of the ACR Preliminary Diagnostic Criteria for Fibromyalgia. J Rheumatol. 2011; 38: 1113-22.

9. Buskila D, Neumann L. Assessing functional disability and health status of women with fibromyalgia: validation of a Hebrew version of the fibromyalgia impact questionnaire. J Rheumatol. 1996; 23: 903-6.

10. Committee for Proprietary Medicinal Products (CPMP). Note for guidance on harmonization of requirements for influenza vaccines. CPMP/BWP/214/96; 12 March 1997. Available from: http://www.cardiff.ac.uk/racdv/resgov/Resources/EMA\%20CFPMP.pdf

11. Hotopf M, David A, Hull L, Ismail K, Unwin C, Wessely S. Role of vaccinations as risk factors for ill health in veterans of the Gulf war: cross sectional study. BMJ 2000; 320: 1363 7.

12. Peakman M, Skowera A, Hotopf M. Immunological dysfunction, vaccination and Gulf War illness. Philos Trans R Soc Lond B Biol Sci. 2006; 361: 681-7.

13. Meroni PL. Autoimmune or auto-inflammatory syndrome induced by adjuvants (ASIA): old truths and a new syndrome? J Autoimmun. 2011; 36: 1-3.

14. Rosenblum H, Shoenfeld Y, Amital H. The common immunogenic etiology of chronic fatigue syndrome: from infections to vaccines via adjuvants to the ASIA syndrome. Infect Dis Clin N Am. 2011; 25: 851-63.

15. Agmon-Levin N, Zafrir Y, Kivity S, Balofsky A, Amital H, Shoenfeld Y. Chronic fatigue syndrome and fibromyalgia following immunization with the hepatitis $B$ vaccine: another angle of the "autoimmune (auto-inflammato- ry) syndrome induced by adjuvants"(ASIA). Immunol Res. 2014; 60: 376-83.

16. Israeli E. Gulf War syndrome as a part of the autoimmune (autoinflammatory) syndrome induced by adjuvant (ASIA). Lupus 2012; 21: 190-4.

17. Allen AD. Is RA27/3 rubella immunization a cause of chronic fatigue? Med Hypotheses 1988; 27: 217-20.

18. Tomljenovic L, Colafrancesco S, Perricone C, Shoenfeld Y. Postural orthostatic tachycardia with chronic fatigue after HPV vaccination as part of the autoimmune/auto-inflammatory syndrome induced by adjuvants - case report and literature review. J Invest Med High Impact Case Rep. 2014; 2: 2324709614527812.

19. Mohammad A, Carey JJ, Storan E, Scarry M, Coughlan RJ, Lee JM. Prevalence of fibromyalgia among patients with chronic hepatitis $\mathrm{C}$ infection: relationship to viral characteristics and quality of life. J Clin Gastroenterol. 2012; 46: 407-12.

20. Buyukkose M, Kozanoglu E, Basaran S, Bayramoglu O, Yarkin F. Seroprevalence of parvovirus B19 in fibromyalgia syndrome. Clin Rheumatol. 2009; 28: 305-9.

21. Adak B, Tekeoğlu Í, Ediz L, Budancamanak M, Yazgan T, Karahocagil K, et al. Fibromyalgia frequency in hepatitis B carriers. J Clin Rheumatol. 2005; 11: 157-9.

22. Wittrup IH, Jensen B, Bliddal H, Danneskiold-Samsoe B, Wiik A. Comparison of viral antibodies in 2 groups of patients with fibromyalgia. J Rheumatol. 2001; 28: 601-3.

23. Rodríguez-Rieiro C, Domínguez-Berjón MF, Vasallodominguez-Berjón E, Cuadrado AR, Carrasco-Garrido P, Jiménez-García R. Coverage and predictors of influenza vaccine uptake among adults aged 16 to 59 years suffering from a chronic condition in Madrid, Spain. Hum Vaccin. 2011; 7: 557-62.

24. Behm FG, Gavin IM, Karpenko O, Lindgren V, Gaitonde S, Gashkoff PA, et al. Unique immunologic patterns in fibromyalgia. BMC Clin Pathol. 2012; 12: 25.

25. Sturgill J, McGee E, Menzies V. Unique cytokine signature in the plasma of patients with fibromyalgia. J Immunol Res. 2014; 2014: 938576.

26. Rodriguez-Pinto I, Agmon-Levin N, Howard A, Shoenfeld Y. Fibromyalgia and cytokines. Immunol Lett. 2014; 161: 200-3. 